Karolinska Institutet

Vivianne Jakobsson

Study Program in Medicine KI

Degree project 30 credits

Fall 2019

Final version

\title{
Resting state connectivity during total sleep deprivation: A functional magnetic resonance study
}

Author: Vivianne Jakobsson 


\title{
Abstrakt
}

\section{Funktionell konnektivitet i vila under total sömnbrist}

Bakgrund: Sömnbrist är ett vanligt problem som kan leda till allvarliga konsekvenser. Funktionell magnetresonanstomografi (fMRI) är en teknik som ofta nyttjas för att undersöka hjärnans nätverk. I denna studie används fMRI för att studera hur konnektiviteten och den globala signalens variabilitet (GSV) i vila ändras vid sömnbrist. Syfte: Undersöka om den globala signalens variabilitet ökar efter sömnbrist. Material och Metoder: denna överkorsningsstudie fick 18 friska deltagare, 20 - 30 år, i randomiserad ordning genomgå 20min funktionell MRI i vila efter en natt normal sömn och efter en sömnlös natt. Vi extraherade den globala signalen och räknade ut standard deviationen för att får den globala signalens variabilitet och analyserade den i förhållande till sömnbrist ja/nej, huvudrörelser, ögon öppna/stängda och försökspersonernas självskattning av sömnighet med Karolinska Sleepiness Scale. Resultat: Den globala signalens variabilitet ökade med sömnbrist $(0.3362 \pm 0.0241, \mathrm{p}<0.0001)$ när vi inte räknade med KSS data. Sömnbrist påverkade inte signifikant när KSS datan var med $(0.0619 \pm 0.1145, \mathrm{p}=0.5889)$. Hög skattning av KSS gav ökad GSV $(0.1497 \pm 0.0409, \mathrm{p}=0.0003)$. GSV ökade även med huvudrörelser $(1.7974 \pm 0.1539, \mathrm{p}<0.0001)$. Det var ingen skillnad på ögon öppna/stängda $(0.0126 \pm 0.0578, \mathrm{p}=0.8278)$, och ingen signifikant ökning för varje tidsperiod av $20 \mathrm{~s} \mathrm{i}$ skannern $(0.0065 \pm 0.0021, \mathrm{p}=0.0029$. Slutsats: Sömnbrist och sömnighet ökar den globala signalens variabilitet. Det går ej att dra mer detaljerade slutsatser angående de olika delarna som bidrar till den globala signalens variabilitet.

\begin{abstract}
Resting state connectivity during total sleep deprivation

Introduction: Sleep deprivation is a common problem that may have serious consequences. In this study, functional magnetic resonance imaging (fMRI), a technique frequently used to study networks in the brain, was used to investigate the resting state of the sleep deprived brain, in order to discover whether this state affects the intrinsic connectivity and the global signal variability (GSV). Aims: To investigate whether GSV increases with sleep deprivation. Material and Methods: In this cross over study 18 healthy participants, age 20 - 30, underwent in randomized order resting-state fMRI for 20min before and after $24 \mathrm{~h}$ sleep deprivation. We extracted the global signal, calculated the standard deviation per participant, and analysed it with respect to sleep depraved yes/no, head motion, eyes open/closed and self-evaluation of sleepiness using Karolinska Sleepiness Score (KSS). Results: We found that GSV was higher during sleep deprivation $(0.3362 \pm 0.0241, \mathrm{p}<0.0001)$ without KSS data. With KSS, sleep deprivation was not significant $(0.0619 \pm 0.1145, \mathrm{p}=0.5889)$. High KSS rating had a significant effect on GSV $(0.1497 \pm 0.0409, p=0.0003)$, as had head motion $(1.7974 \pm 0.1539, p<0.0001)$. There was no significant difference between having eyes open or closed $(0.0126 \pm 0.0578, p=0.8278)$, and no significant increase for each time period of $20 \mathrm{~s}$ in the scanner $(0.0065 \pm 0.0021, \mathrm{p}=0.0029)$. Conclusions: We found that the global signal variation is increased by sleep deprivation and sleepiness. More specific conclusions cannot be made from our data so far.
\end{abstract}

Keywords: "Sleep deprivation", "resting-state", "Global signal variability", "global signal", "fMRI" 


\section{Abbreviations}

ATP - Adenosine Triphosphate

BOLD - Blood Oxygen Level Dependent

CBF - Cerebral Blood Flow

CBV - Cerebral Blood Volume

EEG - Electroencephalogram

FC - Functional Connectivity

fMRI - functional Magnetic Resonance Imaging

GSV - Global Signal Variability

HADS-D - Hospital Anxiety and Depression Scale-depression

ISI - Insomnia Severity Index

KSS - Karolinska Sleepiness Scale

REM - Rapid Eye Movement

rs-fMRI - resting state fMRI 


\section{Introduction}

\section{The function of sleep}

Although sleep accounts for approximately 8h of humans' daily life (1), the reasons why we sleep remain largely unknown. Sleep can be roughly divided into two stages that are alternated and repeated i.e. Non-rapid eye movement (NREM) and rapid eye movement (REM). NREM sleep is associated with low frequency electroencephalogram (EEG) measured waves, i.e. slow-waves, and decreased neuronal activity, while REM sleep onset on the other hand is marked by high frequency waves (2). The exact function of sleep has been investigated and discussed, but a consensus has yet to be reached, and leading theories on why humans and other mammals sleep offer a variety of explanations (3), three of which will be described below.

Early ideas were based on evolutionary advantages of sleep (4). Sleeping allows us to reduce overall energy expenditure (5) during the time when we cannot feed and helps us avoid predators in a rest-activity cycle. In addition, there are claims that the immune system is enhanced by sleep in the healthy as well as the diseased body. Infectious diseases often induce energy-demanding immune responses (6), making it advantageous to conserve energy during disease, i.e. to sleep. Thus, it is suggested that sleep has been evolutionarily conserved due to its energy-sparing fitness benefits. However, these theories leave certain aspects of sleep unexplained, such as loss of environmental awareness and dreaming, both of which often accompany sleep.

Another proposed explanation is that sleep mainly benefits brain metabolism. The brain requires more glucose during wakefulness compared to slow-wave sleep (7). Glucose is needed in the biochemical process that produces adenosine triphosphate (ATP) for use in the $\mathrm{Na}^{2+} / \mathrm{K}^{+}$gates that drive synaptic transmission (8). Porkka-Heiskanen et. al. measured adenosine, a byproduct of brain energy metabolism, in vivo using microdialysis, and proposed that prolonged wakefulness reduces energy supply in the basal forebrain leading to an increase in extracellular adenosine, which is re-phosphorylated during sleep in order to restore brain energy (9). While it would be interesting to directly measure brain tissue ATP during sleep and wake, this has proven to be difficult due to ATP instability in high-oxygen environments $(10)(10)(10)(10)(10)(10)(10)$. Adenosine has been suggested as a hypnogenic 
substance when measured in other animals (11) and the sleepiness-countering effects of adenosine receptor antagonists such as caffeine have been demonstrated (12).

Another proposed function of sleep is that it may serve as a way to adapt and strengthen neural networks. Neurons and glial cells form synapses guided by signaling molecules and gene expressions, many of which are also involved in the regulation of sleep (13). An activity-dependent mechanism creates strong connections and loss of plasticity should there not be a counter-mechanism to curb the positive feedback circle (14). Sleep is suggested to have both constructive and destructive effects on synapses, including function such as consolidating or weakening memories (15), inducing motor and sensory cortex plasticity and increasing synaptic efficiency by downscaling glutamatergic receptors (16). This would explain why unconsciousness is a necessary component of sleep.

\section{Sleep deprivation}

Sleep deprivation is defined as " $A$ disruption and reduction in the number of hours of sleep normally needed by a person "(17). Reasons for loss of sleep are many, and include the stress of having small children, irregular working hours, recreational nightly activities, noisy sleeping environment, an excessive intake of caffeine and sleep disorders $(18,19)$. Such disorders can vary greatly in severity, from relatively harmless acute insomnia to deadly fatal familial insomnia.

Sleep deprivation has negative effects on both somatic and mental health. Diseases such as Type 2 diabetes and obesity are both linked to sleep deprivation, possibly due to the role of the circadian rhythm in glucose metabolism, energy expenditure and appetite regulation (20). In addition, disordered sleep is seen in a number of psychiatric disorders such as schizophrenia, depression and anxiety disorders, as well as in neurodegenerative disorders such as Alzheimer's disease, Huntington's disease and Parkinson's disease (21).

Inadequate sleep has adverse effects on daytime productivity (22) and cognitive performance. When cognitive performance was objectively measured in conjunction with electroencephalography (EEG) markers, test results were worse after sleep deprivation even though test persons' own subjective ratings of their sleepiness and cognitive skills remained largely unchanged (23). Memory formation and consolidation is also negatively affected by 
sleep deprivation (24). Loss of attention, vigilance (25) and motor skills are some of the effects of sleep deprivation. It has been found that prolonged wakefulness of 17-18h causes a drop in response time similar to a blood alcohol concentration of $0.05 \%$ (26). Drowsiness behind the wheel is also associated with impaired risk assessment ability, unfortunately leading to more risk-taking while drowsy in the traffic (27). The National Sleep Foundation Drowsy Driving Consensus Working Group, an expert panel instructed to create a consensus on the subject of drowsy driving, reviewed the evidence from 346 studies and concluded that it is unsafe for otherwise healthy individuals to sleep for less than $2 \mathrm{~h}$ in the preceding $24 \mathrm{~h}$ before driving (28).

\section{Functional Magnetic Resonance Imaging}

Functional magnetic resonance imaging (fMRI) is an imaging technique that visualizes brain activity using brain-oxygen-level-dependent (BOLD) signals. The technique helps to detect brain activity, as oxygenated blood flow is coupled to neural activation (29). Functional connectivity (FC) is the covariance between BOLD signals from different brain areas. If two areas of the brain are seemingly activated together, with or without a known structural connection, they are said to be functionally connected. By measuring FC it is possible to map brain activity during specific tasks, rest or sleep, making the clinical value of fMRI considerable. In order to reduce neurologic deficits after brain tumour resections, fMRI data can be used to identify motor, speech and language areas in addition to or instead of intraoperative mapping (30). Resting state fMRI (rs-fMRI) has been extensively investigated as a possible biomarker for various psychiatric disorders including depression, mood disorders and neurodegenerative such as e.g. Alzheimer's disease and Parkinson's disease (3134). Resting state is assumed when the person being scanned is instructed to fixate their eyes at a point and not perform any particular tasks.

In the search for neural networks associated with states of sleep, wake and arousal, fMRI has been greatly helpful. Tagliazucchi et al used polysomnography EEG in combination with fMRI in order to investigate wakefulness in participants undergoing rs-fMRI and found that a third of participants involuntarily fell asleep within 3 minutes of the resting state scan. (35). This is significant because the onset of sleep alters the functional networks of the brain (36). 


\section{The global signal}

When analyzing rs-fMRI data, spontaneous fluctuations in BOLD signals that can be averaged over the entire brain have been observed as a global signal. Schölvinck et al. performed an experiment using microelectrodes on non-human primates that showed strong positive correlations between amongst spontaneous neural activity and the global signal (37). However, because the BOLD signal is an indirect measure, there are a number of possible confounders in the global signal e.g. cerebral blood volume (CBV), blood flow (CBF), arterial $\mathrm{O}_{2}$ and $\mathrm{CO}_{2}$ ratios, and various other metabolic interactions. These factors combined with other sources of noise e.g. head motion, blood pressure changes, and respiratory cycles make it difficult to separate confounding noise from neural signal (38). There is an ongoing discussion about whether the global signal should be removed in order to reduce noise, or if it should be kept as to not lose potentially valuable data.

Wong et al. described a negative correlation between the global signal amplitude, sometimes also known as the global signal variability (GSV), and EEG measured vigilance before and after ingestion of caffeine (39). Also, previous studies have shown that sleep deprivation decreases the intrinsic connectivity of the brain (40), and increases the global signal variability. This suggests that the relationship between the global signal and wakefulness requires further investigation.

Consequences of sleep deprivation are well documented, but the underlying physiology remains only partially discovered. Total sleep deprivation is likely to make subjects more vulnerable to involuntary sleep. Thus, while there are no direct clinical applications of this project, it will provide an opportunity to further study the global signal variability and its relationship with sleep deprivation.

\section{The Stockholm Sleepy Brain Project}

This project is a part of a collaboration between Stockholm University and Karolinska Institute and part 3 in a series of studies called Stockholm Sleepy Brain. Sleepy Brain 1 (SB1) produced a multimodal brain imaging dataset of younger (20-30) and older (65-75) participants after normal asleep and during sleep deprivation. Results from this study was later used for hypothesis generation for studies that came to follow. Sleepy Brain 2 (SB2)(41) 
also used both younger and older participants and found that GSV increases during a $3 \mathrm{~h}$ partial sleep deprivation intervention.

\begin{abstract}
Aim
The aim of this study was to determine whether the global signal variability would increase after sleep deprivation.
\end{abstract}

\title{
Materials and methods
}

\section{Study design}

The study was designed as a cross-over study comparing $24 \mathrm{~h}$ total sleep deprivation and habitual sleep. Total sleep deprivation was chosen as partial sleep deprivation was investigated during SB2 (41) with data available for comparison.

Participants acted as their own controls and were randomized to which order they would undergo the interventions. Each participant completed three scanning sessions, once for the baseline scan, once after a night of total sleep deprivation, and once after a night of habitual sleep. fMRI imaging took place in the morning at 07.30 after Participants stayed at our sleep lab during sleep deprived night under supervision.

\section{Participants}

18 participants were recruited through posters at university campus sites. Before the baseline scan, they were asked to complete a set of questionnaires designed to determine if they were suitable participants for the project. Prospective participants with ferromagnetic implants or claustrophobia were excluded due to MR incompatibility. As we wanted healthy participants, prospective participants with health issues including sleep apnea, hypertension, diabetes and history of heart surgery were excluded. Pregnant women were excluded. Prospective participants with a Hospital Anxiety and Depression Scale-Depression (HADS-D) score over 8 and Insomnia Severity Index (ISI) over 15 were excluded. Hospital Anxiety and Depression scale (HADS) (42) is a self-assessment scale designed for detecting depression, anxiety and mood disorders in patients, in an outpatient context. In this study, the depression scale (HADS-D) was used to exclude participants scoring 8 or higher as depression might affect their response to the emotion regulation task. Insomnia Severity Index (ISI) (43) is a 
screening assessment of insomnia. We used an ISI score over 15 as an exclusion criterion to reduce individual differences in habitual sleep time in the "full sleep" intervention. Prospective participants with a caffeine intake of more than four cups of coffee daily, psychoactive medication use, and habitual nicotine use were excluded as these may affect sleeping habits.

Participants were required to be between 20-30 years old as age has been shown to affect functional connectivity and we compared results between total and partial sleep deprivation. Participants were also required to have normal eyesight, be fluent in Swedish, have a habitual bedtime between 22.00-01.00, and live in the greater Stockholm area.

We included data from our pilot study during November 2018 and March 2019 in our statistical analyses. The pilot differed from the final study design in that participants used an actigraph at home for monitoring the sleep deprived night instead of staying at our sleep lab.

\section{Experiment design}

Each imaging session was completed in approximately one hour. Three tasks were given to the participants in this study; the first was a sustained attention task, the second an emotional regulation task, and finally a resting state task. This project only includes the resting state task, thus only this task will be reported here.

The resting state task lasted for in total 20 minutes. While in the scanner, participants were instructed to simply look at a fixation cross for 5 minutes and then close their eyes for the remaining 15 minutes. An eye-tracker was used to observe whether participants' eyes were open or closed. If eyes were closed for more than 5 seconds during the first 5 minues, we presumed the participant had fallen asleep and the experiment leader spoke a wake-up call. Respiratory frequency, heart rate and electrocardiography (ECG) were monitored as these factors are known to contribute to the global signal and thus needed to be taken into account during analysis.

Participants were asked to self-evaluate their sleepiness between each task in total four times using the Karolinska Sleepiness Scale (KSS), a nine-point Likert-type scale similar in validity to objective measures of sleepiness (44) where $<5$ is considered sleepy, $>5$ is considered not sleepy and 5 is neutral. Results of KSS was used to estimate wake/sleep state. 


\section{Data acquisition}

A 3 T GE 750 MRI scanner was used to acquire images. T1-weighted structural images were obtained with 180 slices and used as anatomical reference. Settings for fMRI image acquisition was interleaved starting at the bottom, 40 slices, with 2 seconds between each slice. Global signal was determined from fMRI images of from a mask including the gray matter of the brain.

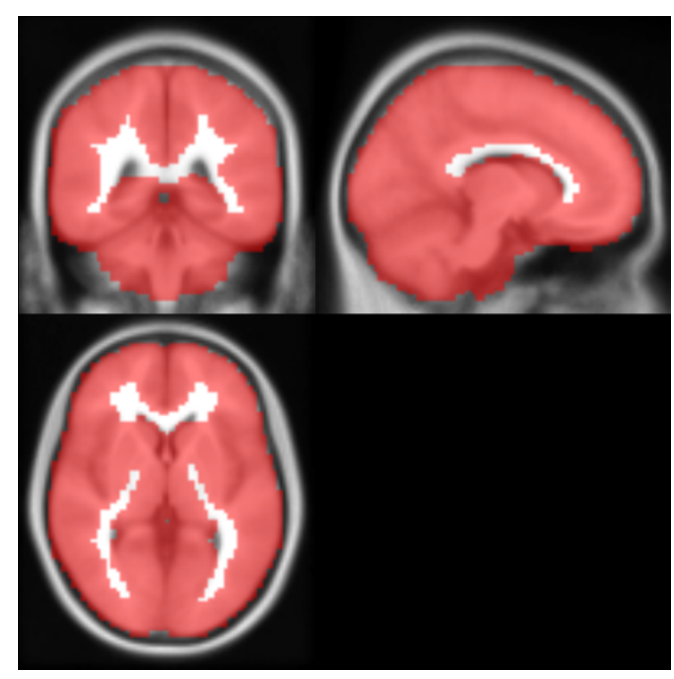

Figure 1: A mask of the gray matter is applied to fMRI images and used to identify the global signal.

\section{Preprocessing}

All images were preprocessed using a standard pipeline using fMRIprep (45).

Functional images and structural images are preprocessed separately using a standard pipeline and the co-registered. Co-registration makes it possible to present fMRI results with greater detail as structural images are more detailed and serve as anatomical reference.

Preprocessing of structural images is done by normalization. This is done using a series of linear and non-linear transformations applied to each image to make them fit a standard brain model (atlas template) made by the Montreal Neurological Institute. This is done in order to 
be able to generalize results over a population and identify the same areas of activity in different people.

Preprocessing of functional images involves several steps. Functional images are often treated as if they were taken at the same time, while there are actually milliseconds in between slices. This would be a source of error. If one were to compare the images created from sampled slices at different time stamps and superpose them onto one another, it would not be a perfect fit. To correct for this, one performs a slice time correction by choosing a reference slice, often in the beginning or the middle of the sequence and shifting the other slices to match this. Head motion is another potential source of error as voxels may be slightly displaced between each slice in the sequence. Realignment reduces this error by performing a series of transformations on each slice to align it with the previous slice in the sequence. As the functional variability is not adjusted during normalization, spatial smoothing is applied and reduces the signal-to-noise ratio by averaging data points to compensate for functional variability.

\section{Statistical methods}

Analysis of data was performed using $\mathrm{R}$ with the nmle package. Variability of the global signal was measured using the log-transformed standard deviation of the time series, in blocks of 20 seconds. A mixed-effects model was used, in order to correctly account for repeated measures within participants, to test the hypothesis that global signal variability is greater after sleep deprivation.

We entered sleep deprivation condition, eyes open/closed, KSS score and time on task as fixed effects. We entered participant identification as a random effect. $\mathrm{P}<0.05$ was considered significant.

\section{Ethical considerations}

Participation in this study was fully voluntary, and information was given and consented to before participation. Any potential harm to participants was limited and easily prevented by strict adherence to the exclusion criteria, as fMRI is a well-tested and safe imagining technique. Possible adverse reactions to fMRI included claustrophobia and anxiety, which none of participants reported. Any side effects related to sleep deprivation were reversed by catch-up sleep the next day. In order to prevent traffic accidents due to sleep deprivation all 
sleep deprived participants was offered a taxi ride to the MR center. One participant suffered minor skin burns from EOG electrodes.

Few studies have investigated the effects of sleep deprivation on functional networks in the brain and how they are related to perceived sleepiness and influence on certain neurological functions. Very small risk of harm was posed to participants in this study compared to the overall contribution to research and potential future benefits to the general population.

All images were fully anonymized for use in the study. Images were also linked into the participants' individual medical journals for their own benefit, as any incidental findings on the images would have been examined by a neuroradiologist and further investigated.

This study was approved by Regionala etiksprövningsnämnden i Stockholm, dnr 2012/1098$31 / 2$.

\section{Results}

\section{Participants}

A total of 18 participants, 10 males and 8 females, were included from both the pilot and SB3 (see tables 1 and 2). 10 participants from SB3 completed all scanning sessions (fig. 2).

Table 1: General characteristics of participants, Sleepy Brain 3

\begin{tabular}{|l|c|c|}
\hline Parameter & Mean & $\begin{array}{c}\text { Standard } \\
\text { deviation }\end{array}$ \\
\hline Age & 26 & \pm 2.8 \\
\hline Height/cm & 173 & \pm 14 \\
\hline Weight/kg & 69 & \pm 14 \\
\hline HADS-D & 2.6 & \pm 0.9 \\
\hline ISI & 4.5 & \pm 3.0 \\
\hline
\end{tabular}

HADS-D = Hospital Anxiety and Depression Scale - Depression ISI= Insomnia Severity Index 
Table 2: General characteristics of participants, Sleepy Brain 2

\begin{tabular}{|l|c|c|}
\hline Parameter & Mean & $\begin{array}{c}\text { Standard } \\
\text { deviation }\end{array}$ \\
\hline Age & 29 & \pm 6 \\
\hline Height/cm & N/A & N/A \\
\hline Weight/kg & 69.9 & \pm 11.9 \\
\hline HADS-D & 7.3 & \pm 1.9 \\
\hline ISI & 9 & \pm 2.1 \\
\hline
\end{tabular}

HADS-D $=$ Hospital Anxiety and Depression Scale - Depression ISI= Insomnia Severity Index

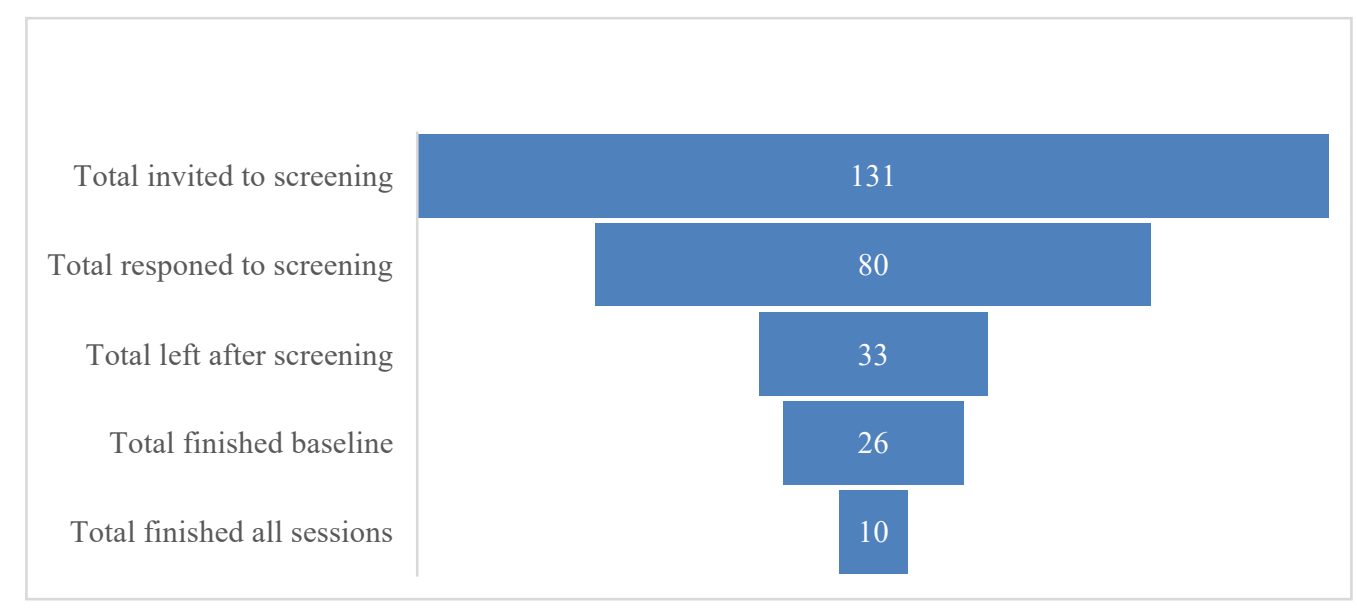

Figure 2: Chart of participants, excluding those from our pilot study.

\section{fMRI Quality control}

A quality check using MRIQC was manually done after pre-processing. We controlled field of view to make sure the whole brain is visible in the images. We checked co-registration between structural and functional images to (fig. 3) and corrected for head motion through framewise displacement. Signal from each tissue compartment and per voxel was manually examined (fig. 4). All images were reasonably good quality and suitable for analysis. 


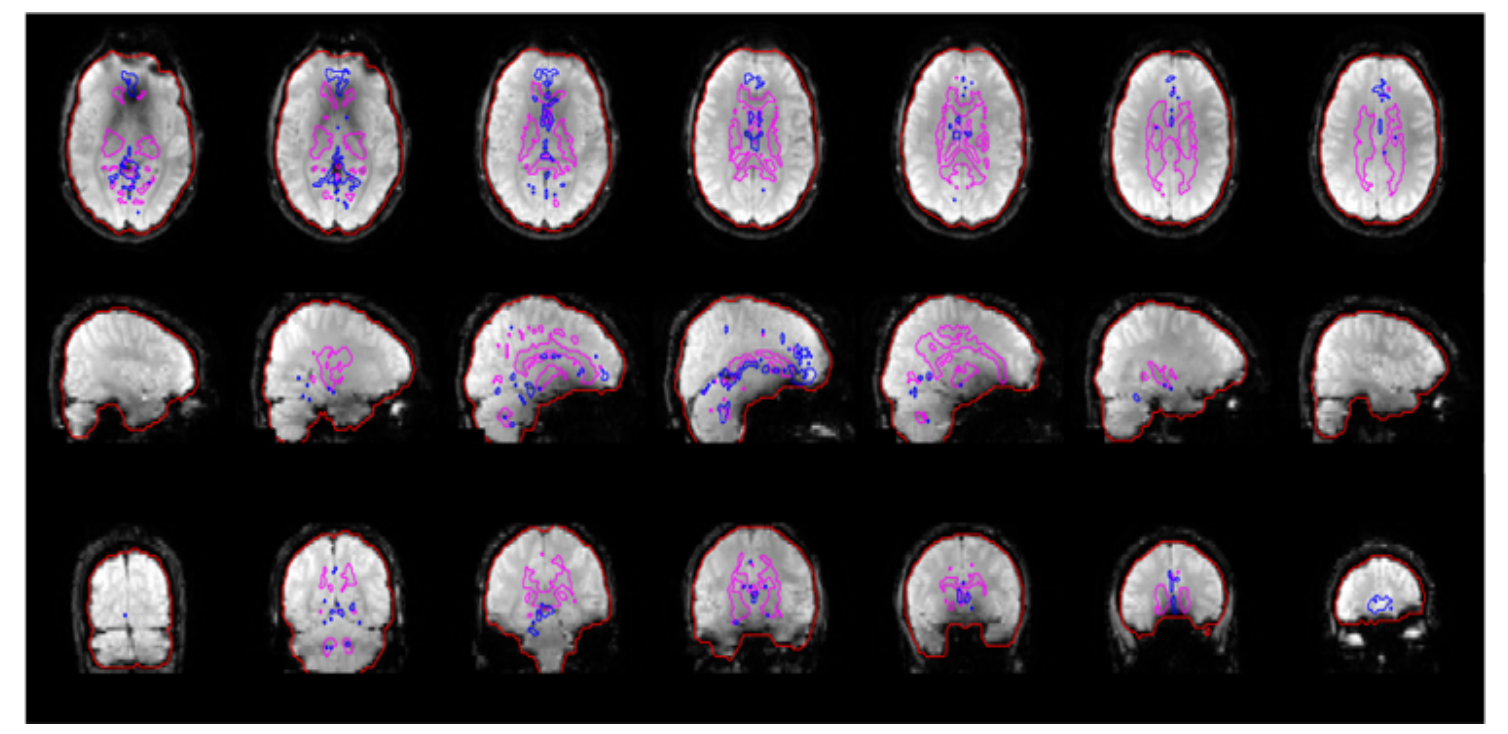

Figure 3: Example of fMRI quality control of co-registration. fMRI images in three planes. Anatomical reference structures are marked in purple and blue.

\section{Confounding variables}

We did not manage to obtain reliable output from equipment monitoring heart rate, ECG and respiratory frequency and thus could not adjust GSV in relation to these factors. There was a significant effect of head motion on GSV as described below.

\section{fMRI Results}

After applying our regression model to the signal time series, we found that the global signal variation was higher during total sleep deprivation $(0.3362 \pm 0.0241, \mathrm{p}<0.0001)$ before we factored in KSS data. With KSS, sleep deprivation lost significance $(0.0619 \pm 0.1145$, $\mathrm{p}=0.5889)$. High KSS rating had a significant effect on GSV $(0.1497 \pm 0.0409, \mathrm{p}=0.0003)$. In addition, GSV increased with head motion $(1.7974 \pm 0.1539, \mathrm{p}<0.0001)$. 


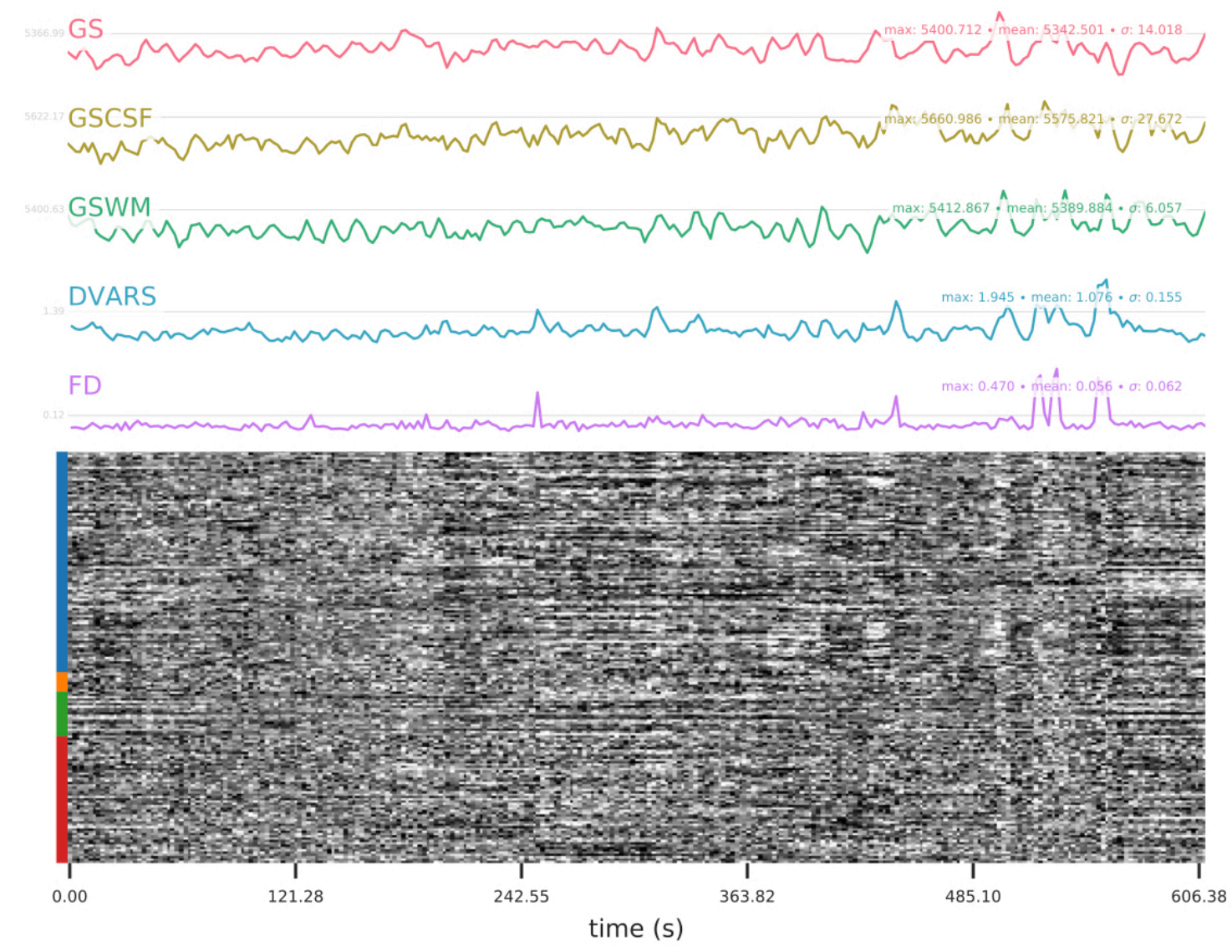

Figure 4: fMRI summary plot.

GS - global signal; average signal from gray matter

GSCSF - global signal within anatomical mask of cerebrospinal fluid (CSF)

GSWM - global signal within anatomical mask of white matter

DVARS - derivative of root mean squared (RMS) variance over voxels

FD - framewise displacement; calculated bulk movements due to head movements

The carpet plot at the bottom shows a summary of BOLD signals. Blue area shows cortical signals, orange area show subcortical signals, green area shows cerebellar signals, red area show signals from white matter and CSF.

There was no significant difference between having eyes open or eyes closed $(0.0126 \pm$ $0.0578, \mathrm{p}=0.8278$ ), and no significant increase for each time period of $20 \mathrm{~s}$ in the scanner $(0.0065 \pm 0.0021, \mathrm{p}=0.0029$. Figure 5 shows GSV during sleep deprived sessions compared to habitual sleep. 


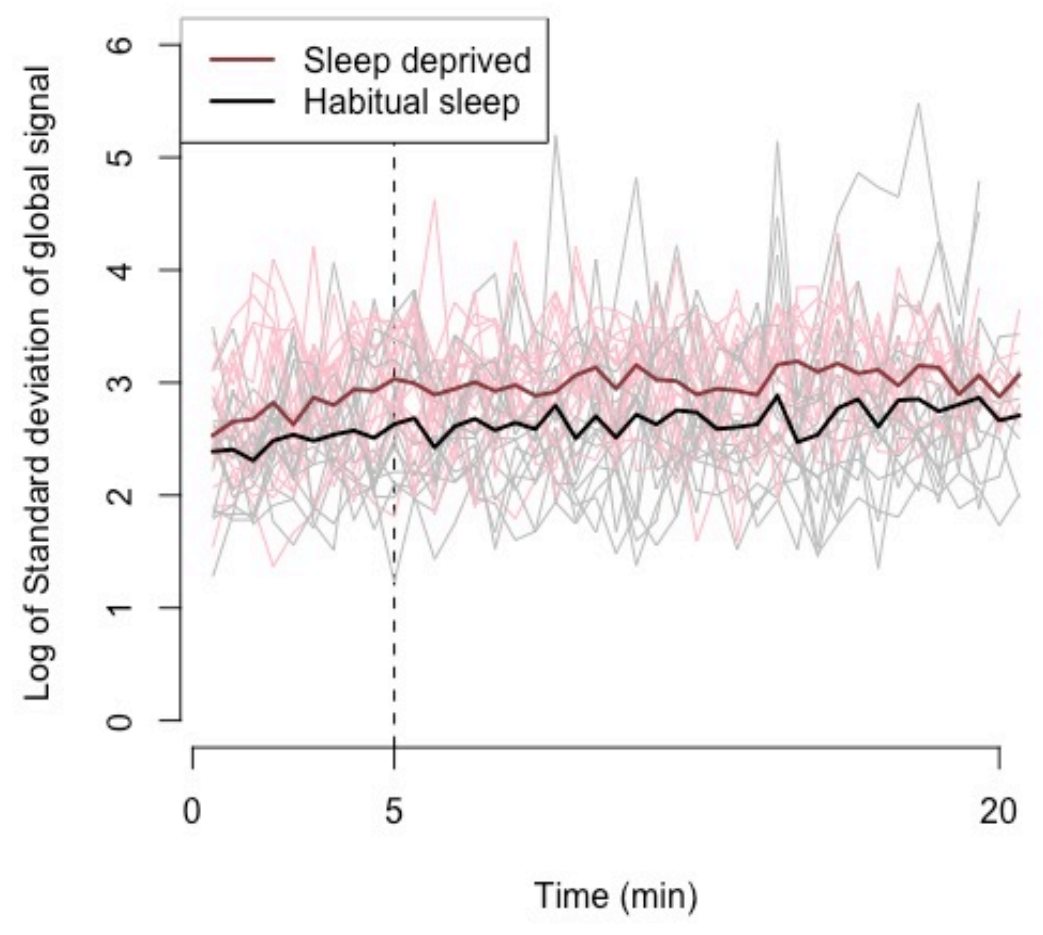

Figure 5: Global signal variability (GSV) over time. The red line shows mean GSV for all participants during sleep deprivation. The black line shows mean GSV for all participants after habitual sleep.

Self-evaluated sleepiness using KSS was used as an estimate for wake/sleep state. Sleep deprivation did not cause a significant increase in KSS score during the resting state experiment $(1.1222 \pm 0.6522, \mathrm{p}=0.1110)$ (fig. 6$)$. 


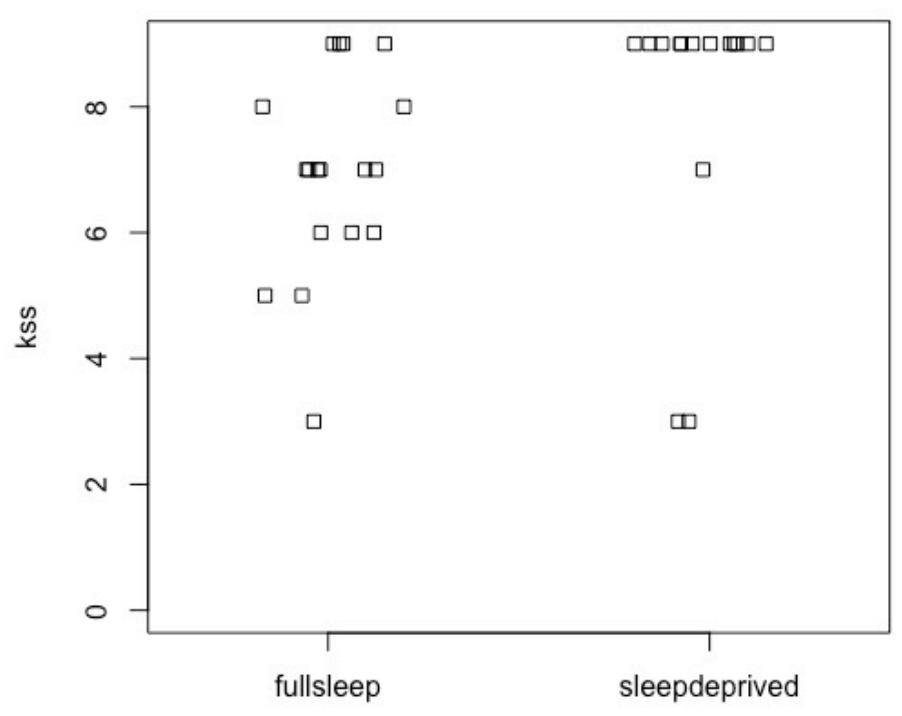

Figure 6: Karolinska Sleepiness Score (KSS) plotted against sleep condition.

\section{Discussion}

\section{Recapitulation and discussion}

In the present study we analyzed GSV from resting state fMRI data after habitual sleep and after $24 \mathrm{~h}$ total sleep deprivation. GSV is the variation of the average BOLD signal from gray matter. Because global signal reflects changes in not only neural networks but also cerebral blood flow and head motion, it is frequently viewed as a source of noise and filtered out before data analysis. There is an ongoing discussion on whether removing global signal is beneficial or if valuable information is deleted in doing so. Our results showed that sleep deprivation had a significant effect on GSV unless subjective sleepiness, rated using KSS, was factored in. This suggests that sleepiness may mediate the relationship between sleep deprivation and GSV. While KSS ratings did not significantly increase during sleep deprivation, this may be due to the limited sample, as a non-significant effect in the expected direction was observed. While we cannot prove that sleep deprivation increased GSV through subjective sleepiness, this is a possible mechanism. Sleep deprivation for more than $24 \mathrm{~h}$ is likely to increase subjective sleepiness and thus GSV.

However, our results agree with previous results from SB2 (46) using partial sleep 
deprivation, as we did not use KSS in the same way as in that study. It also agrees with the work by Wong et al. (47) that showed that GSV decreases with caffeine intake and increased vigilance, and with the findings by Fukunuga et al. (48) that GSV increases during sleep. GSV is thus suggested to play a role in vigilance and degree of wakefulness. However, it is important to note that BOLD signals are an indirect measure of brain activity affected by all nervous activity including sympathetic activity in peripheral nerves that could lead to changes in cerebral blood flow. Correlations between GSV and degree of sleepiness may very well be due to confounders such increased heart rate, higher blood pressure or head motion which tend to be more frequent during sleep deprivation (49). We intended to measure heart rate and respiratory frequency but the output from our equipment was not reliable. It is therefore reasonable to speculate that the increase in GSV during sleep deprivation may be due to wake-state instability, but more research is needed before any more detailed conclusions can be made.

Wake-state instability, or as described by Tagliazucchi et al., the tendency to drift between wakefulness and sleep (35), has been shown to increase when investigated using resting state fMRI. EEG remains the gold-standard for monitoring sleep stages, but fMRI in combination with EEG is difficult from both technical and safety perspectives and requires special equipment. As we did not need the additional temporal resolution provided by EEG, we originally intended to monitor participants' wakefulness using EOG, with slow eye movements indicating onset of sleep and blinking indicating arousal. This effort was abandoned after an incident in which one of the participants suffered minor skin burns from electrodes placed in the face. Subjecive sleepiness using KSS is similar in validity to objective methods for evaluating sleepiness and wake/sleep state, making it a reasonable substitute for EOG data (44). We did use an eye-tracker to ensure participants did not fall asleep during the first 5 minutes of the resting state experiment, but we had no way of knowing whether they were asleep during the eyes-closed remaining 15 minutes until the next KSS evaluation.

\section{Strengths and limitations}

The main weakness of this study is the small sample size which greatly limits statistical power and validity. External validity is also limited by the homogeneity of our participants, most of which were university students. In our previous study using partial sleep deprivation 
we chose two different age groups, younger (20-30 years old) and older (50-65 years old). In this study we only chose younger participants as this increases internal validity and minimizes "healthy volunteer bias" as diseases tend to have a higher prevalence amongst older people.

Strengths include using total sleep deprivation instead of partial sleep deprivation as this is assumed to increase the effect on the outcome compared to our previous study, thus increasing our chances of finding a real effect. As is the case with all cross over studies, participants act as their own controls, excluding interindividual differences. Sleep deprivation was monitored by use of an actigraph or by letting participants stay one night supervised in our sleep lab, ensuring that no participants accidentally fell asleep during sleepless nights.

\section{Significance}

We investigated sleep deprivation using fMRI instead of traditional EEG. Both fMRI and EEG are major non-invasive techniques used to investigate sleep and sleep deprivation. fMRI has a greater spatial resolution and provides more precise localization of activated brain regions during tasks. EEG on the other hand is a direct measure of neuronal activation and has a better temporal resolution compared to BOLD signals received using fMRI. In order to better understand the functions and dynamics of sleep, EEG and fMRI combined would complement each other and provide data with superior spatiotemporal resolution (50).

However, not all sleep and vigilance related connectivity found using fMRI are mapped, and the role of the global signal has yet to be fully understood. Studying the functions involved in maintaining wakefulness or inducing sleep by analysing resting state fMRI of sleep deprived subjects might supply insight into the neurophysiology of sleep and the consequences of sleep deprivation. Whether or not the global signal should be regarded as a source of noise has been a subject of discussion amongst fMRI researchers, but the results from this study in agreement with previous studies indicate that the global signal, itself perhaps not as much as its variability, might be of value when studying wake and sleep.

Our results are not representative of the general population as the sample size of this study was small and our participants were within a narrow range of ages. Only healthy participants were included, results may not be generalizable to those with psychiatric disorders or 
neurodegenerative diseases. Results are however equally applicable to both sexes as age, but not sex, is assumed to have an effect on functional connectivity.

There are no clinical applications of this part of the study for the moment. However, considering that sleep deprivation is a common problem, further research remains of interest to the general population.

\section{Future studies}

In future studies of wake-state instability using resting state fMRI, wake and sleep should be monitored with EEG or more careful use of EOG in order to increase significance of results. It would be interesting to include different age groups to investigate how they would differ in global signal variation during sleep deprivation, as older people tend to have less functional connectivity (41). This study is continued in the Stockholm Sleepy Brain Project with a larger sample size.

A hypothesis that could be generated from this study to be investigated in the future is that total sleep deprivation has a greater effect on global signal variability compared to partial sleep deprivation.

\section{Conclusions}

We conclude that the global signal variation is increased by sleep deprivation. The effect could be due to increased blood flow, increased head motion, and changes in brain connectivity due to wake-state instability.

\section{Contributions}

Our research group consisted of Gustav Nilsonne, Paolo d'Onofrio, Sandra Tamm, Torbjörn Åkerstedt, Mats Lekander and Jimmy Hermansson. MRI preprocessing was done by Joe Wexler and Gustav Nilsonne. Fellow medical student Poyan Karimi and I used the same data in our projects; we both assisted with data collection, contributed to data analysis and supervised participants in the sleep lab. 


\section{Acknowledgements}

I am grateful for having had the opportunity to briefly be part of the Stockholm Sleepy Brain project and would like to thank all members of the research group for their support. I would like to especially thank my supervisor Gustav Nilsonne for his patient guidance throughout this project, Jimmy Hermansson and Paolo d'Onofrio for their help in gathering data.

\section{Supervisor}

Gustav Nilsonne MD, PhD, Researcher

Karolinska Institutet, Department of Clinical Neuroscience

Stockholm University, Stress Research Institute

Mail: gustav.nilsonne@ki.se

\section{References:}

1. C H, N G, N M. Recent Evidence on

Worldwide Trends on Sleep Duration. Current Sleep Medicine Reports. 2015;1(4):9.

2. Harris CD. Neurophysiology of sleep and wakefulness. Respir Care Clin N Am. 2005;11(4):567-86.

3. Siegel JM. Clues to the functions of mammalian sleep. Nature. 2005;437(7063):126471.

4. Rechtschaffen A. Current perspectives on the function of sleep. Perspect Biol Med. 1998;41(3):359-90.

5. Walker JM, Haskell EH, Berger RJ, Heller HC. Hibernation at moderate temperatures: a continuation of slow wave sleep. Experientia. 1981;37(7):726-8.

6. Baracos VE, Whitmore WT, Gale R. The metabolic cost of fever. Can J Physiol Pharmacol. 1987;65(6):1248-54.

7. Heiss WD, Pawlik G, Herholz K, Wagner R, Wienhard K. Regional cerebral glucose metabolism in man during wakefulness, sleep, and dreaming. Brain Res. 1985;327(1-2):362-6. 8. Thomas RC. Electrogenic sodium pump in nerve and muscle cells. Physiol Rev. 1972;52(3):563-94.

9. Porkka-Heiskanen T, Alanko L, Kalinchuk A, Stenberg D. Adenosine and sleep. Sleep Med Rev. 2002;6(4):321-32.

10. Heller HC. Repeatability Is Not the Same as Accuracy. Sleep. 2011;34(7).

11. Radulovacki M, Virus RM, Djuricic-Nedelson M, Green RD. Adenosine analogs and sleep in rats. J Pharmacol Exp Ther. 1984;228(2):268-74.

12. Fredholm BB, Bättig K, Holmén J, Nehlig A, Zvartau EE. Actions of caffeine in the brain with special reference to factors that contribute to its widespread use. Pharmacol Rev. 1999;51(1):83-133.

13. Impey S, Obrietan K, Storm DR. Making new connections: role of ERK/MAP kinase signaling in neuronal plasticity. Neuron. 1999;23(1):11-4. 
14. Kavanau JL. Sleep and dynamic stabilization of neural circuitry: a review and synthesis. Behav Brain Res. 1994;63(2):111-26.

15. Stickgold R, Walker MP. Sleep-dependent memory triage: evolving generalization through selective processing. Nat Neurosci. 2013;16(2):139-45.

16. Liu ZW, Faraguna U, Cirelli C, Tononi G, Gao XB. Direct evidence for wake-related increases and sleep-related decreases in synaptic strength in rodent cortex. J Neurosci. 2010;30(25):8671-5.

17. Kent M. The Oxford Dictionary of Sports Science \& Medicine 3ed: Oxford University Press; 2006.

18. Clark I, Landolt HP. Coffee, caffeine, and sleep: A systematic review of epidemiological studies and randomized controlled trials. Sleep Med Rev. 2017;31:70-8.

19. Boivin DB, Boudreau P. Impacts of shift work on sleep and circadian rhythms. Pathol Biol (Paris). 2014;62(5):292-301.

20. Knutson KL, Spiegel K, Penev P, Van Cauter E. The metabolic consequences of sleep deprivation. Sleep Med Rev. 2007;11(3):163-78.

21. Wulff K, Gatti S, Wettstein JG, Foster RG. Sleep and circadian rhythm disruption in psychiatric and neurodegenerative disease. Nat Rev Neurosci. 2010;11(8):589-99.

22. Rosekind MR, Gregory KB, Mallis MM, Brandt SL, Seal B, Lerner D. The cost of poor sleep: workplace productivity loss and associated costs. J Occup Environ Med. 2010;52(1):918.

23. Van Dongen HP, Maislin G, Mullington JM, Dinges DF. The cumulative cost of additional wakefulness: dose-response effects on neurobehavioral functions and sleep physiology from chronic sleep restriction and total sleep deprivation. Sleep. 2003;26(2):11726.

24. M. W. Sleep, memory and brain plasticity. Sleep Medicine. 2007;8(59).

25. J. L, D. D. Sleep deprivation and Vigilant Attention. Annals of the New York Academy of Sciences. 2008;1129(1):17.

26. Williamson AM, Feyer AM. Moderate sleep deprivation produces impairments in cognitive and motor performance equivalent to legally prescribed levels of alcohol intoxication. Occup Environ Med. 2000;57(10):649-55.

27. McKenna BS, Dickinson DL, Orff HJ, Drummond SP. The effects of one night of sleep deprivation on known-risk and ambiguous-risk decisions. J Sleep Res. 2007;16(3):245-52.

28. Czeisler CA, Wickwire EM, Barger LK, Dement WC, Gamble K, Hartenbaum N, et al. Sleep-deprived motor vehicle operators are unfit to drive: a multidisciplinary expert consensus statement on drowsy driving. Sleep Health. 2016;2(2):94-9.

29. Glover GH. Overview of functional magnetic resonance imaging. Neurosurg Clin N Am. 2011;22(2):133-9, vii.

30. Jiménez de la Peña M, Gil Robles S, Recio Rodríguez M, Ruiz Ocaña C, Martínez de Vega V. Cortical and subcortical mapping of language areas: correlation of functional MRI and tractography in a 3T scanner with intraoperative cortical and subcortical stimulation in patients with brain tumors located in eloquent areas. Radiologia. 2013;55(6):505-13.

31. Heller AS, Johnstone T, Shackman AJ, Light SN, Peterson MJ, Kolden GG, et al. Reduced capacity to sustain positive emotion in major depression reflects diminished maintenance of fronto-striatal brain activation. Proc Natl Acad Sci U S A. 2009;106(52):2244550.

32. Lee MH, Smyser CD, Shimony JS. Resting-state fMRI: a review of methods and clinical applications. AJNR Am J Neuroradiol. 2013;34(10):1866-72.

33. Badhwar A, Tam A, Dansereau C, Orban P, Hoffstaedter F, Bellec P. Resting-state network dysfunction in Alzheimer's disease: A systematic review and meta-analysis. Alzheimers Dement (Amst). 2017;8:73-85. 
34. Wolters AF, van de Weijer SCF, Leentjens AFG, Duits AA, Jacobs HIL, Kuijf ML. Resting-state fMRI in Parkinson's disease patients with cognitive impairment: A meta-analysis. Parkinsonism Relat Disord. 2019;62:16-27.

35. Tagliazucchi E, Laufs H. Decoding wakefulness levels from typical fMRI resting-state data reveals reliable drifts between wakefulness and sleep. Neuron. 2014;82(3):695-708.

36. Horovitz SG, Braun AR, Carr WS, Picchioni D, Balkin TJ, Fukunaga M, et al. Decoupling of the brain's default mode network during deep sleep. Proc Natl Acad Sci U S A. 2009;106(27):11376-81.

37. Schölvinck ML, Maier A, Ye FQ, Duyn JH, Leopold DA. Neural basis of global restingstate fMRI activity. Proc Natl Acad Sci U S A. 2010;107(22):10238-43.

38. Murphy K, Birn RM, Bandettini PA. Resting-state fMRI confounds and cleanup. Neuroimage. 2013;80:349-59.

39. Wong CW, Olafsson V, Tal O, Liu TT. The amplitude of the resting-state fMRI global signal is related to EEG vigilance measures. Neuroimage. 2013;83:983-90.

40. De Havas JA, Parimal S, Soon CS, Chee MW. Sleep deprivation reduces default mode network connectivity and anti-correlation during rest and task performance. Neuroimage. 2012;59(2):1745-51.

41. Nilsonne G, Tamm S, Schwarz J, Almeida R, Fischer H, Kecklund G, et al. Intrinsic brain connectivity after partial sleep deprivation in young and older adults: results from the Stockholm Sleepy Brain study. Sci Rep. 2017;7(1):9422.

42. Zigmond AS, R.P. S. The Hospital Anxiety and Depression Scale. Acta Psychiatrica Scandinavica. 1983;67(6):9.

43. Bastien $\mathrm{CH}$, Vallières A, Morin CM. Validation of the Insomnia Severity Index as an outcome measure for insomnia research. Sleep Med. 2001;2(4):297-307.

44. Akerstedt T, Anund A, Axelsson J, Kecklund G. Subjective sleepiness is a sensitive indicator of insufficient sleep and impaired waking function. J Sleep Res. 2014;23(3):240-52.

45. Esteban O, Markiewicz CJ, Blair RW, Moodie CA, Isik AI, Erramuzpe A, et al. fMRIPrep: a robust preprocessing pipeline for functional MRI. Nat Methods. 2019;16(1):1116.

46. Tamm S, Nilsonne G, Schwarz J, Lamm C, Kecklund G, Petrovic P, et al. The effect of sleep restriction on empathy for pain: An fMRI study in younger and older adults. Sci Rep. 2017;7(1):12236.

47. Wong CW, DeYoung PN, Liu TT. Differences in the resting-state fMRI global signal amplitude between the eyes open and eyes closed states are related to changes in EEG vigilance. Neuroimage. 2016;124(Pt A):24-31.

48. Fukunaga M, Horovitz SG, van Gelderen P, de Zwart JA, Jansma JM, Ikonomidou VN, et al. Large-amplitude, spatially correlated fluctuations in BOLD fMRI signals during extended rest and early sleep stages. Magn Reson Imaging. 2006;24(8):979-92.

49. Kato M, Phillips BG, Sigurdsson G, Narkiewicz K, Pesek CA, Somers VK. Effects of sleep deprivation on neural circulatory control. Hypertension. 2000;35(5):1173-5.

50. Babiloni F, Mattia D, Babiloni C, Astolfi L, Salinari S, Basilisco A, et al. Multimodal integration of EEG, MEG and fMRI data for the solution of the neuroimage puzzle. Magn Reson Imaging. 2004;22(10):1471-6. 